\title{
Racetrack-shape fixed field induction accelerator for giant cluster ions
}

\author{
Ken Takayama, ${ }^{1,2,3,4}$ Toshikazu Adachi, ${ }^{1,2}$ Masayoshi Wake, ${ }^{1}$ and Katsuya Okamura, ${ }^{1,2}$ \\ ${ }^{1}$ High Energy Accelerator Research Organization (KEK), Tsukuba, Ibaraki 305-0801, Japan \\ ${ }^{2}$ The Graduate University for Advanced Studies (SOKENDAI), Hayama, Kanagawa 240-0193, Japan \\ ${ }^{3}$ Tokyo Institute of Technology, 4259 Nagatsuda, Kanagawa 226-8503, Japan \\ ${ }^{4}$ Tokyo City University, Setagaya, Tokyo 158-8557, Japan
}

(Received 8 January 2014; published 8 May 2015)

\begin{abstract}
A novel scheme for a racetrack-shape fixed field induction accelerator (RAFFIA) capable of accelerating extremely heavy cluster ions (giant cluster ions) is described. The key feature of this scheme is rapid induction acceleration by localized induction cells. Triggering the induction voltages provided by the signals from the circulating bunch allows repeated acceleration of extremely heavy cluster ions. The given RAFFIA example is capable of realizing the integrated acceleration voltage of $50 \mathrm{MV}$ per acceleration cycle. Using $90^{\circ}$ bending magnets with a reversed field strip and field gradient is crucial for assuring orbit stability in the RAFFIA.
\end{abstract}

DOI: 10.1103/PhysRevSTAB.18.050101

PACS numbers: 29.20.D-, 81.05.ub, 96.50.Dj

\section{INTRODUCTION}

Since 1994, when CNRS-IN2P3 at Orsay demonstrated acceleration of cluster ions to a high energy by using a tandem accelerator, the new field of condensed matter science has rapidly evolved, focusing on nonlinear irradiation effects on target materials, such effects arising from the extremely high energy density of cluster ions [1]. To date, electrostatic accelerators have been the only means of accelerating cluster ions. The achievable maximum voltage of such electrostatic accelerators is limited by discharge effects. In addition, acceleration of highly charged ions in a tandem accelerator is not expected. The limit is empirically known to be, at most, around 20-30 MV [2]. Acceleration of cluster ions beyond this level is not actually possible. For instance, the Orsay tandem accelerator is nominally operated at 14.5 MV for acceleration of C-60. It seems obvious that repeated acceleration in a circular accelerator will be an efficient method to obtain higher energy cluster ions. Unfortunately, conventional circular accelerators, such as rf synchrotrons and cyclotrons, are not available for this purpose because of limitations on frequency bandwidths. The mass ratio to charge state, that is, $A / Q$, is of the order of $10^{2}-10^{4}$ for cluster ions. The revolution frequency in a circular ring varies across a wide range during one acceleration cycle. Its dynamic range is far beyond the capability of the conventional rf technology that integrates a resonant cavity and rf amplifier. As a matter of fact, there is no presently known way to accelerate $\mathrm{U}^{1+}$.

As a novel circular accelerator employing induction acceleration, the induction synchrotron has been developed

Published by the American Physical Society under the terms of the Creative Commons Attribution 3.0 License. Further distribution of this work must maintain attribution to the author(s) and the published article's title, journal citation, and DOI. at KEK since it was first proposed [3]. The concept of the induction synchrotron was demonstrated as a type of slow cycling synchrotron in 2006 [4]. Recently, a small-scale induction synchrotron, called the KEK digital accelerator, where heavy ions are directly injected into the ring from a $200 \mathrm{kV}$ ion source, has been successfully used to accelerate heavy ions [5]. Ions in an induction synchrotron are accelerated and captured by pulse voltages generated by the 1-to-1 pulse transformers known as "induction acceleration cells" (ICs). ICs are energized through the corresponding switching power supply (SPS), in which solid-state power devices are employed as switching elements and their turning to the on/off state is operated through gate signals that are digitally manipulated by the signal from a circulating ion beam. Consequently, acceleration synchronized to the revolution of the ion beam is always guaranteed, regardless of the type of ions and their possible charge state. Here, any ions directly injected from an ion source can be accelerated from an extremely low speed to almost the speed of light. In this context, the acceleration of cluster ions in the KEK digital accelerator has been considered since the early days of its construction [6]. However, it turns out that the acceleration of cluster ions in the KEK digital accelerator does not yield desirable characteristics. This is understood by using a parameter $V$ for the equivalent acceleration voltage of an electrostatic accelerator, which is defined in terms of atomic mass unit $m$, mass number $A$ and charge state $Q$ of a cluster ion, maximum flux density $B$, and bending radius $\rho$ in a circular accelerator ring:

$$
\begin{aligned}
V[\text { volt }]= & \left(\frac{A}{Q}\right) \cdot \frac{m c^{2}}{e} \\
& \cdot\left\{\sqrt{1+\left[\left(\frac{Q}{A}\right) \cdot\left(\frac{e}{m c^{2}}\right) \cdot c \cdot B \rho\right]^{2}}-1\right\} .
\end{aligned}
$$


As an example, $\mathrm{C}-60(A=720, Q=7)$ is considered. Substituting machine parameters of the KEK digital accelerator $(B=1.1 \mathrm{~T}$ and $\rho=3.3 \mathrm{~m})$, we have $V=6 \mathrm{MV}$. This magnitude is insufficient for applications that need to explore far beyond $V=14.5 \mathrm{MV}$. Of course, by using a gigantic synchrotron, such as Tevatron, RHIC, or LHC, we can expect an extremely large value of $V$. However, the laboratories operating these high-energy accelerators have high-energy physics as their mission and are supported by academics who are not interested in high-energy cluster ions. Thus, a compact circular accelerator capable of providing attractive high-energy giant cluster ions is still desired and should become a priority for future development of cluster ion accelerators.

For the purpose of obtaining high-energy cluster ions in a relatively compact accelerator, there are three possible scenarios: (i) small induction synchrotron with superconducting guiding magnets of $8.5 \mathrm{~T}$; (ii) induction sector cyclotron; (iii) racetrack-shape fixed field induction accelerator.

In scenario (i), high-energy cluster ions are obtained because of an extremely large value for $B$; in scenarios (ii) and (iii), the ions are obtained by increasing the bending radius $\rho$. Here, it must be pointed out that scenarios (i) and (ii) present a particular obstacle: rapid ramping of the superconducting magnet is difficult, and stability in low field regions is not assured because variability among magnets may exceed the acceptance. In addition, a large aperture is required because of the large beam emittance associated with low-energy injection. This makes it difficult to practically realize the $8.5 \mathrm{~T}$ magnet. Scenario (ii) (an induction sector cyclotron) has been seriously considered, and an example of such a device is given in Ref. [7]. The preliminary design shows that an induction sector cyclotron of a practical size can provide high-energy cluster ions at attractive energies. However, requirements on the induction acceleration cell are quite stringent because of the wide range of orbit shifts. The cross section of an induction acceleration cell is necessarily a large racetrack shape with a $2 \mathrm{~m}$ inner aperture, and this demands a large volume of magnetic material core and a difficult manufacturing process that includes winding thin magnetic ribbon tape into the racetrack shape. This is not impossible, but it is very difficult. In scenario (iii), a RAFFIA has one or two straight sections, in which the beam orbit never changes from injection to extraction. The region can accommodate induction acceleration cells, which are similar to compact cells for the induction synchrotron with a toroidal shape [8]. This scenario does not require any technological leaps. It is noted that a geometrical shape of the RAFFIA is very close to that of a recirculating microtron, although a microwave linac in the recirculating microtron is replaced by a stack of induction acceleration cells in the RAFFIA.

Thus, it is interesting to further explore the possibility of a RAFFIA as a circular accelerator for cluster ions. First, the difference between an electron microtron and heavy-ion RAFFIA is described. Then, an outline of a possible
RAFFIA is briefly explained, focusing on the necessary hardware. Stability in transverse motion may be a key feature of the RAFFIA. This issue will be discussed in detail from the viewpoint of beam physics.

\section{CONCEPTS OF THE RAFFIA}

Historically, a concept of resonance electron accelerator named as "electron cyclotron" was given by Veksler in 1944 [9] and after World War II a resonant electron accelerator similar to the electron cyclotron was independently proposed by Ito and Kobayashi [10], Schwinger [11], and Schiff [12]. The first such type of accelerator was built in Canada in 1948 [13] and named "microtron", since its accelerating cavity was fed by a microwave generator. Early works on microtrons until the 1950s had been reviewed by Roverts [14]. Since the 1970s, electron microtrons, a step beyond the classical microtron for nuclear physics, have been designed and constructed around the world. These microtrons are not a circular accelerator but a kind of recirculator with electron linacs occupying the straight sections. In most cases of electron microtron, the number of orbits is $20-30$ or at most 100 , depending on machine and bending magnet size. Since a large energy gain per turn is provided by an electron linac, a turn separation in the orbit transition region is sufficiently large to allow the installation of either guiding quadrupole magnets or orbit correction magnets. In contrast, the energy gained during induction acceleration in a RAFFIA is limited to $50 \mathrm{kV}$ per turn in practical cases, as seen later. Consequently, the orbit transition region cannot accommodate anything. In electron microtrons, it is vital to maintain synchronization between the bunch revolution and accelerating microwaves because of the discrete jump in orbit length. In a RAFFIA, synchronization is maintained by the intrinsic nature of pulse voltage triggers at arbitrary timing and by monitoring of the circulating ion bunch. Changes in the path length with acceleration never affect synchronization, and so it is not necessary to dwell on this point.

There are two types of RAFFIAs considered here. RAFFIAs with $180^{\circ}$ bending magnets and $90^{\circ}$ bending magnets are illustrated in Figs. 1(a) and 1(b), respectively. Obviously, the $180^{\circ}$ bending magnet version is not practical as a high-energy machine because the size of bending magnet is quite large. Therefore, the $90^{\circ}$ bending magnet RAFFIA will be discussed. This type of electron microtron, which is called a $1.5 \mathrm{GeV}$ harmonic double sided microtron, was constructed at Mainz University [15] and is running now. The structure of the RAFFIA is simple. Four bending magnets each give an orbit bend of $90^{\circ}$. The upper and lower straight sections are occupied by an injection system, extraction system, guiding magnet sets, induction acceleration cells, beam diagnostic system, and orbit correction systems. Straight sections on the right and left 


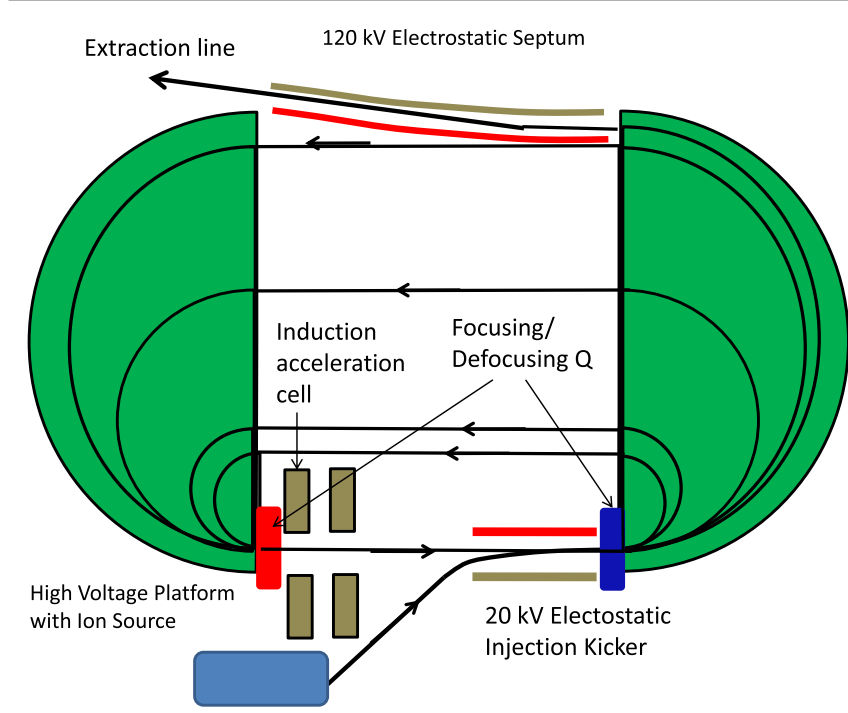

(a)

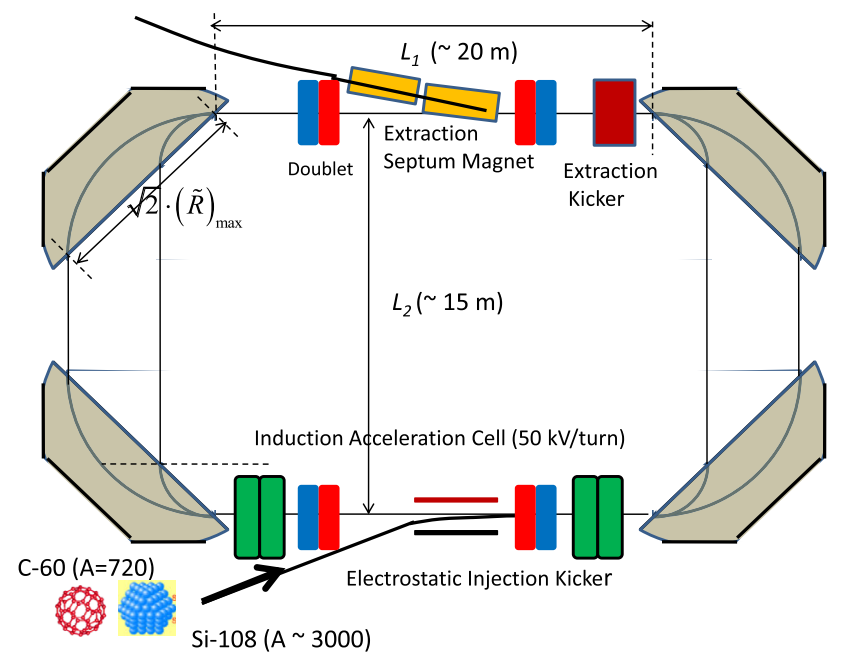

(b)

FIG. 1. (a) RAFFIA with 180 degree bending magnets. (b) RAFFIA with 90 degree bending magnets with the front opening length of $\sqrt{2} \cdot(\tilde{R})_{\max }$, where $\tilde{R}$ is the effective bending radius.

sides are the orbit transition region, which is located in the flat and wide vacuum chamber.

The most serious issue with this RAFFIA is edge defocusing in the vertical direction at the entrance and exit of the bending magnets. Injection and extraction at a $45^{\circ}$ angle produces large defocusing effects and fringing effects. It is crucial to compensate for these effects by introducing additional focusing techniques to a quadrupole doublet system, which is symmetrically placed in the straight sections. For this purpose, a field gradient in the bending magnet and a reversed field strip in front of the bending magnet [16] have been studied. In the stability analysis, all focusing and defocusing effects generated in the quadrupole doublet system and the bending magnets are taken into account.

\section{ION BEAM PARAMETERS AND ACCELERATOR COMPONENTS}

For simplicity, we assume $\mathrm{C}-60(A / Q=720 / 7)$ as a cluster ion here because it is typical and has been used in numerous and varied experiments. In addition, the fixed flux density of the bending magnets is assumed to be $1.5 \mathrm{~T}$. An integrated accelerating voltage of $50 \mathrm{MV}$ is assumed, which determines the size of the RAFFIA.

\section{A. Cluster ion beam and accelerator parameters}

Highly charged C-60 ions will be generated in the laser ion source, which has been demonstrated in many institutes. As a matter of fact, ionization up to $Q=12$ is reported in Ref. [17], although its lifetime was short. Such an ion source is embedded in the $200 \mathrm{kV}$ high voltage platform. Ion beams will be chopped by any longitudinal chopper, such as the Einzel lens chopper [18,19], so as to meet the bunch length required for the RAFFIA. The ion bunch is guided to the RAFFIA through the low energy beam transport line (LEBT) by including an appropriate momentum analyzer and charge-state selector. The bending radius in the bending magnet from beginning of acceleration to end is given as a function of turn number in Fig. 2, where the bending magnetic fields are assumed to be uniform for simplicity.

The maximum bending radius is $\rho=7 \mathrm{~m}$. This implies that a bending magnet with a front edge size more than $2 \rho \sin (\pi / 4)=9.9 \mathrm{~m}$ is required. Its sagitta $\rho[1-\cos (\pi / 4)]$
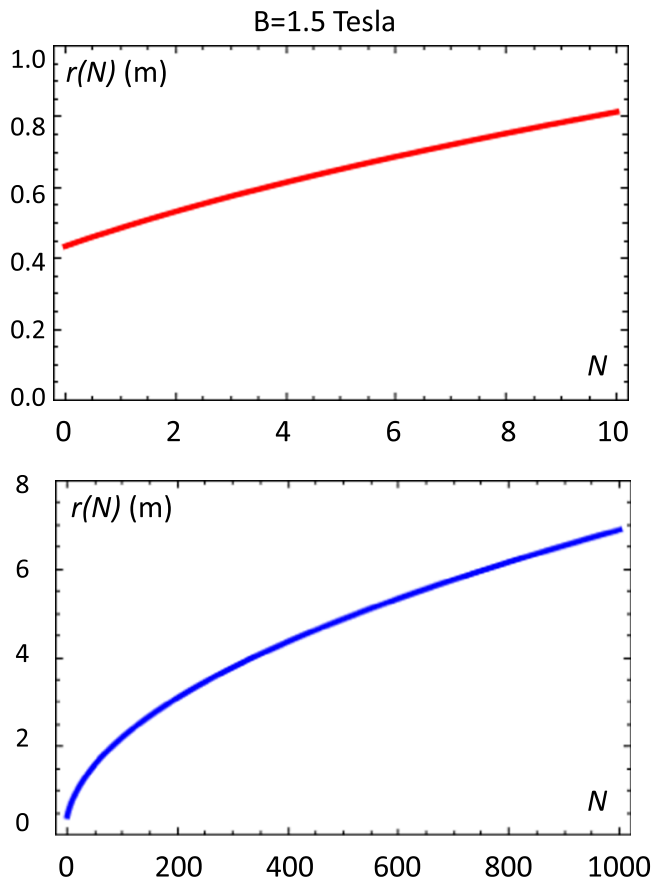

FIG. 2. The bending radius vs turn, from 1st turn to 10th turn (top) and from 1st turn to 1000th turn (bottom). 
TABLE I. Specification of the electrostatic injection kicker.

\begin{tabular}{lc}
\hline \hline Voltage $(\mathrm{kV})$ & $\sim 20$ \\
Electrodes gap/length $(\mathrm{cm})$ & $20 / 80$ \\
Number of interim electrodes & 3 \\
Charging time period $(\mathrm{ms})$ & 20 \\
Charging system & $\mathrm{PFN}$ \\
Turn-off time period $(\mu \mathrm{s})$ & $<1$ \\
Turn-off switch & SI-thyristor matrix array \\
Maximum carrying current $(\mathrm{A})$ & 400 \\
\hline \hline
\end{tabular}

at the maximum bending radius is $2.05 \mathrm{~m}$. As expected, it is quite large. Thus, parameters $L_{1}$ and $L_{2}$ in Fig. 1 that characterize the geometrical feature of the RAFFIA are chosen to be 20 and $15 \mathrm{~m}$, respectively.

\section{B. Injection system}

An electrostatic kicker may be the best choice for oneturn injection. Because the required electrostatic voltage simply scales from the initial acceleration voltage of $200 \mathrm{kV}$, the electrostatic injection kicker at the KEK digital accelerator [20] is suitable for the present purpose. Its parameters are listed in Table I.

To avoid any residual-electric-field effects on the ion bunch completing the first turn after injection, turning off the electrostatic voltage on the electrode is crucial. A thyratron is popular for this purpose. Recently, it has been demonstrated that a conventional thyratron can be replaced by a matrix array of SI-thyristors [21]. It is noted that this solid-state device, which has a high voltage resistance of $4 \mathrm{kV}$ and a high carrying current of $400 \mathrm{~A}$ and fast turningon time similar to that of the thyratron, has a much longer lifetime and is very inexpensive. An example of the injection kicker is shown in Figs. 3(a), 3(b), and 3(c) together with the turning-off profile, driven by the conventional thyratron and SI-thyristor matrix array.

\section{Bending magnet}

The bending magnets are the most crucial components of the RAFFIA, as mentioned earlier. These magnets are extremely large and have a field gradient and reversed field strip in front of the pole edge to stabilize the ion orbits, as discussed in the next section. It is noted that the orbit stability is not guaranteed without the reverse fields in our preliminary study. Schematic views near the magnet front edge are shown in Figs. 4(a) and 4(b). The characteristics of edge focusing are illustrated as is the orbit on the median plane.

The reversed field in front of the pole edge gives negative bending, which yields strong focusing effects in the vertical direction and, remarkably, mitigates defocusing effects at the main magnet entrance because of the shallow injection angle. This is why the reversed fields are employed.

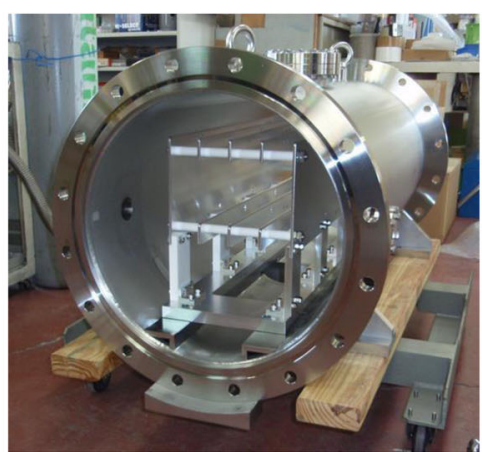

(a)
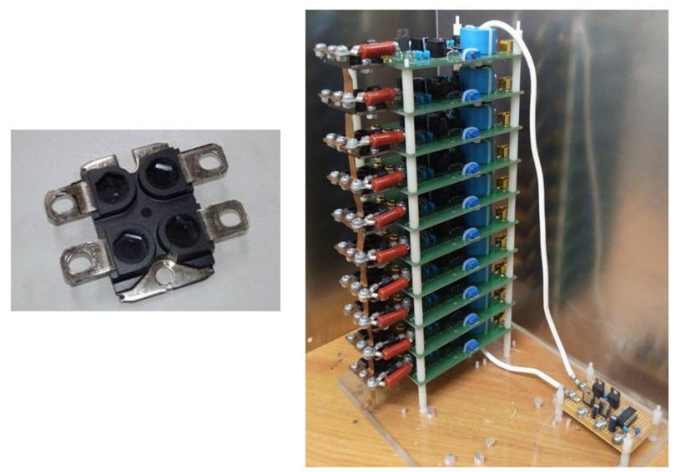

(b)

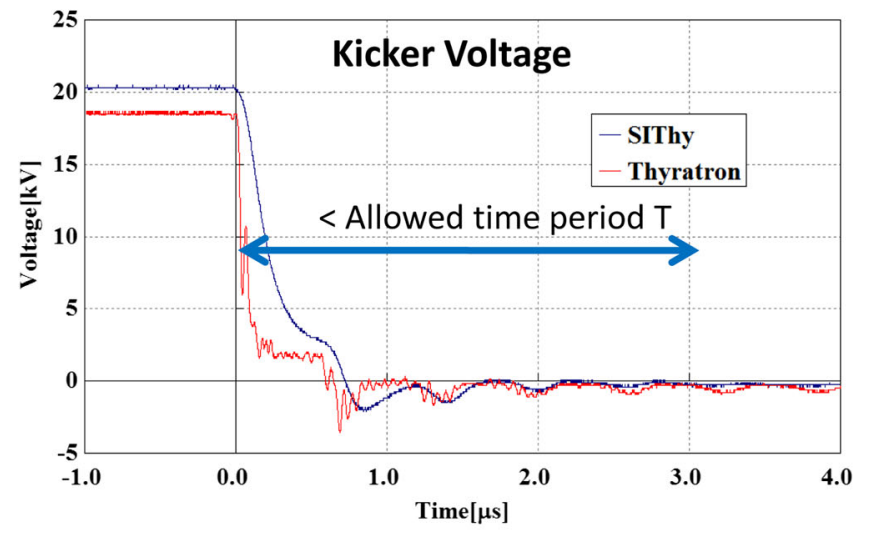

(c)

FIG. 3. (a) The electrostatic injection kicker. (b) SI-thyristor (made in Shindengen) and its matrix array. (c) Time profile in voltage turning-off process.

The magnetic field distribution of a typical bending magnet $B_{y}(X)$ is shown in Fig. 5, where $\mathrm{X}$ is the coordinate perpendicular to the magnet pole edge on the median plane.

\section{Focusing magnets (doublet)}

The doublet itself is both trivial and standard. However, it is excited by a programmed power supply, synchronized with acceleration, because the required field gradient has to be carefully controlled to secure orbit stability in the horizontal and vertical directions. The overall duty factor of the RAFFIA may be determined from the capability of 


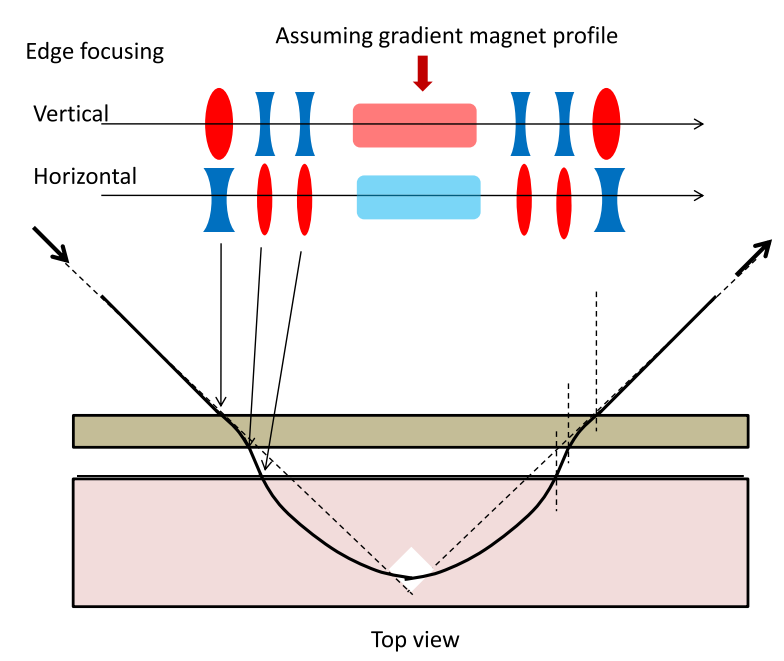

(a)

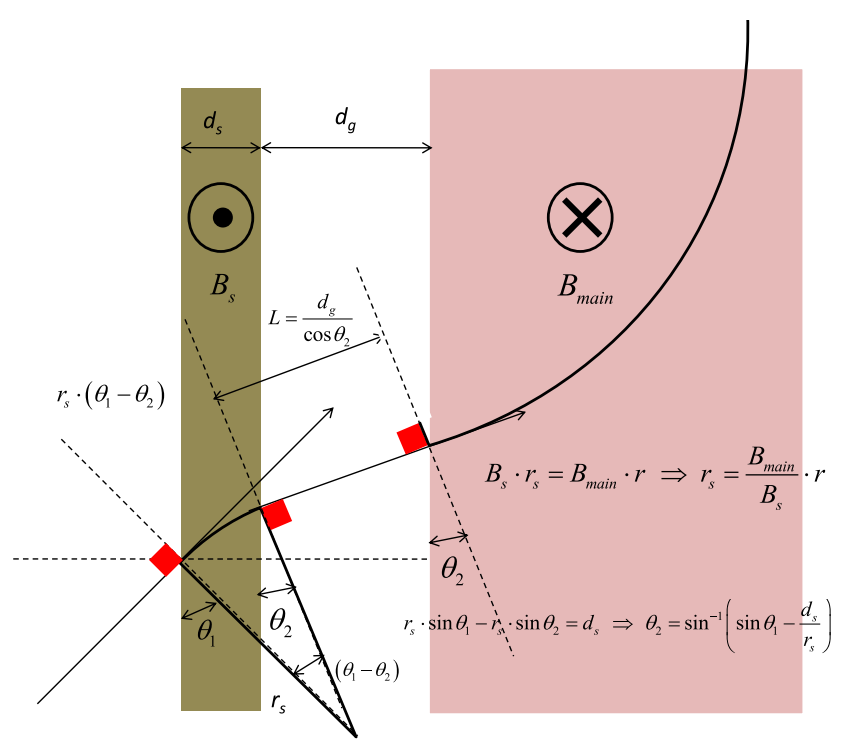

(b)

FIG. 4. (a) Top view of the bending magnet with characteristics of the edge focusing. Red and blue in the upper figure implies focusing and defocusing, respectively. (b) Orbit geometry near the magnet entrance, where rectangular magnetic field profiles with the uniform flux density of $B_{s}$ and $B_{\text {main }}$ are assumed.

the power supply of the focusing magnet, since there are no other limitations on the acceleration cycle.

\section{E. Induction acceleration system}

In the demonstration of a slow cycling induction synchrotron using the KEK $12 \mathrm{GeV}$ synchrotron and in the demonstration of a fast cycling induction synchrotron by the KEK digital accelerator, an induction acceleration system consisting of a small toroidalshape IC and SPS with a full-bridge circuit structure driving the IC has been utilized. The SPS employed 28 power metal-oxide-semiconductor field effect transistors

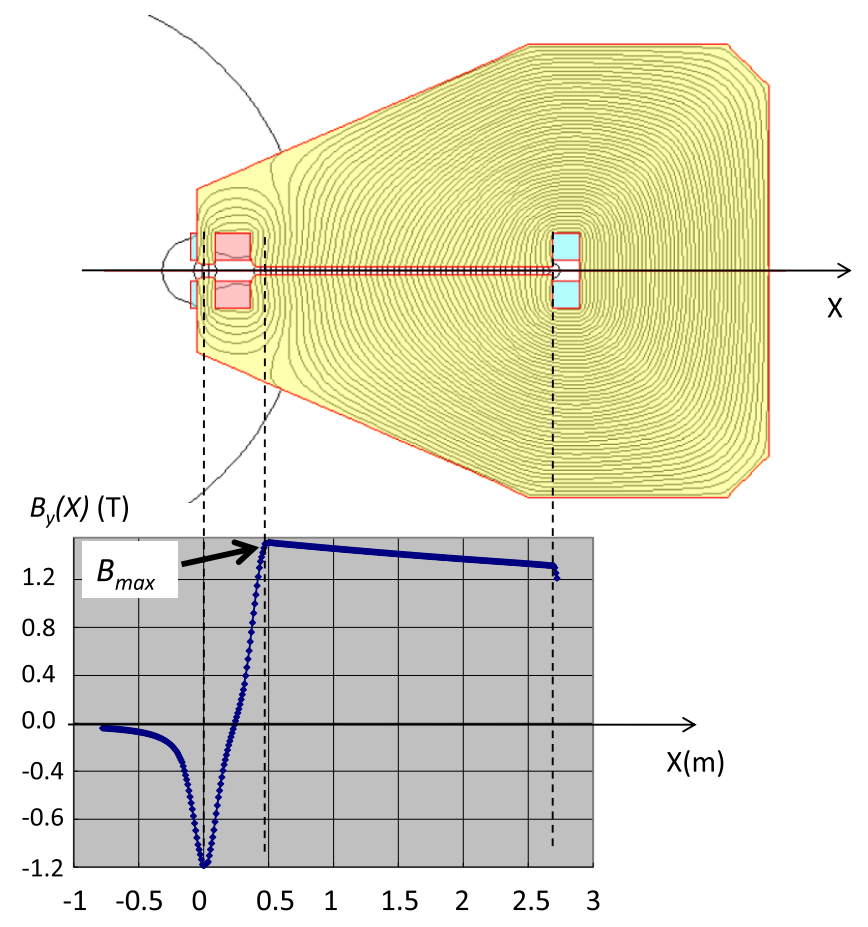

FIG. 5. Cross section of the bending magnet with gradient and reversed field strip and $B_{y}(X)$, where $B_{\max }$ is the maximum flux density in the field distribution.

(MOSFETs) (four arms with seven elements each) as switching elements [22]. The trigger module divides the master trigger signal into 28 isolated signals and the divided and transferred light gate signals are sent to individual MOSFET boards. Recently, a novel SiC-JFET package with higher voltage resistance and larger carrying current capability has been developed [23]. In the near future, we will be able to demonstrate a $2.4-3.0 \mathrm{kV}$ $\mathrm{SiC}$ device. If this package is employed, a simple and small SPS will be available for the RAFFIA. An

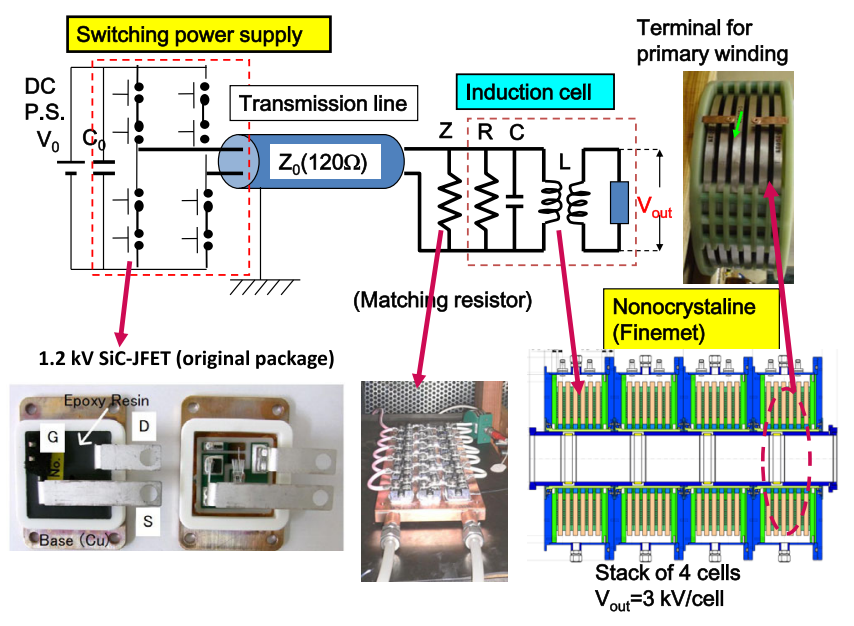

FIG. 6. Equivalent circuit of the induction acceleration cell and their key components. 


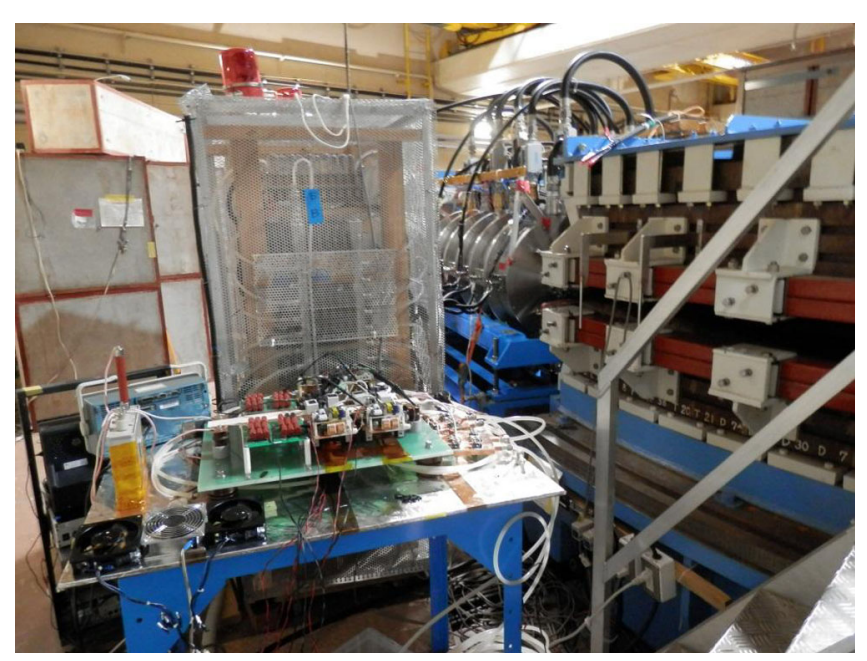

FIG. 7. Test stand to realize the SPS employing the SiC-JFET devices, where the full bridge circuit is mounted on the flat board in the center and the induction cells on the straight section and the bending magnet of the KEK digital accelerator are visible in the right-hand side.

equivalent circuit of the induction acceleration system is shown in Fig. 6. A test of the existing IC by the SPS employing the first generation of $\mathrm{SiC}$ devices has been successfully carried out [24] and the test bench is shown in Fig. 7.

\section{F. Vacuum}

In general, heavy ions have large cross sections of electron capture in the low-energy region and electron stripping in the higher-energy region. An extremely good vacuum level of $10^{-8} \mathrm{~Pa}$ must be realized. A major factor determining the vacuum will be outgassing from the large metal surface of the bending region and the orbit transient region. The pumping system and inner-surface conditioning will be crucial to realize the required vacuum level. It is reported that extremely heavy cluster ions, such as albumin ( $A=66,400, Q=38$ ) in an electrostatic storage ring, have a long lifetime of tens of seconds under a good vacuum of $10^{-9} \mathrm{~Pa}[25]$.

A large flat vacuum chamber must be installed in the magnet poles and in the dispersive region between the two magnet pairs. The chambers in the magnets should be fixed at the pole shoes inside the magnet gap. Outside the magnets, the chambers must be mechanically sustained to prevent them from imploding.

\section{G. Extraction system}

The extraction system, consisting of the extraction kicker and septum magnet (a familiar component in many synchrotrons), can be simple for single-turn extraction (fast extraction). For multiturn extraction (slow extraction), a high-voltage wire septum device is available as a combination of one-half integer and one-third integer resonant extraction magnets. An abundance of experience has been obtained with these in synchrotrons [26].

\section{H. Monitoring of beam position and orbit correction}

There is some concern about errors in the integrated bending field and local inhomogeneity in the bending field. These error fields cause something like a closed orbit distortion (COD) observed in a usual synchrotron. Such CODs must be corrected by an orbit correction system integrating the beam centroid monitors and correction magnets. Orbit separation in conventional microtrons is rather large and a correction magnet on an individually separated beam line can be equipped [27]. However, the turn by turn COD correction is unrealistic in the RAFFIA because of an almost continuous change in the orbit. It is quite important to minimize error components. Fortunately, the bending magnet is static. Shims may be useful for error correction of the integrated bending field. Meanwhile, current sheets to compensate the local inhomogeneity, which are installed in the pole gaps, should be helpful, as demonstrated in the MAMI project [27]. Beam position monitors in the bending magnet region and the dispersive region between the two magnet pairs may be an array of segmented electrostatic position monitors for the vertical direction and a multilaser wire monitor for the horizontal direction [28]. R\&D works for these monitor systems must be carried out for their realization.

\section{STABILITY IN THE TRANSVERSE DIRECTION}

For simplicity, the present RAFFIA is regarded as a synchrotron with a variable circumference and bending magnet length. Under this assumption, we define an instantaneous equilibrium orbit on the median plane and betatron oscillation in the horizontal and vertical directions, where the concept is justified as well as in other fixed field machines such as a cyclotron and fixed field alternating gradient synchrotron.

\section{A. Focusing and defocusing in the bending magnet and reversed field strip}

Focusing and defocusing components are intrinsic in flat $90^{\circ}$ bending magnets. It is trivial and well known that edge focusing and defocusing are dominant in both directions because of the large entrance and exit angles. They are listed in Table II in Appendix A.

Preliminary calculations indicate that there is no stable solution in the vertical direction for the combination of doublets and the above focusing and defocusing parameters of the flat bending magnet. So, a linear field gradient and reversed field strip, as seen in Figs. 4(a) and 4(b), are introduced in the bending magnet. Both effects can remarkably improve stability in the vertical direction. The amount of gradient and the amplitude of the reversed 
field are parameters to be optimized. Here, $\left[d B_{y}(X) / d X\right] / B_{\max }=-0.1 \mathrm{~m}^{-1}$ and $B_{\text {reverse }}=-0.8 \cdot B_{\max }$ are assumed.

\section{Field gradient in the bending magnet}

For simplicity, a uniform field gradient in the direction perpendicular to the magnet pole front edge, $d B_{y}(X) / d X(=$ constant $)$ is assumed. The beam orbit in the bending magnet is necessarily extended, as seen in Fig. 8, where $Z$ is the coordinate parallel to the magnet pole front edge on the median plane. In circular or recirculating accelerators it is usual to take one coordinate (orbit coordinate) $s$ along the equilibrium orbit. The field gradient $d B_{y}(s) / d x$ must be defined along this orbit. The relationship between $d B_{y}(X) / d X$ and $d B_{y}(s) / d x$ is described below.

The orbit in terms of the Cartesian coordinate $(X, Z)$ in the bending magnet is written by $Z=Z(X)$.

The field gradient along the path is given in the form of $\quad \frac{\partial B_{y}(s)}{\partial x}=\frac{\partial B_{y}(X)}{\partial X} \cdot \cos \phi \quad$ From $\quad s=v_{s} \cdot d t \quad$ and $\cos \phi=\frac{d Z / d t}{v_{s}}=\frac{d Z}{d s}$, we have the $k$-value along the orbit,

$$
K(s)=-\frac{1}{B \rho} \cdot \frac{\partial B_{y}(s)}{\partial x}=-\frac{1}{B \rho} \cdot \frac{\partial B_{y}(X)}{\partial X} \cdot \frac{d Z}{d s} .
$$

Here, $v_{s}$ is the longitudinal velocity along $s$ and $B \rho$ is the magnetic rigidity of an ideal particle. $\phi$ is the angle between Cartesian coordinate $X$ and the horizontal coordinate $x$ perpendicular to $s$, and this varies as a function of $s$. A typical example of orbits and $d Z / d s$ is shown in Fig. 8.

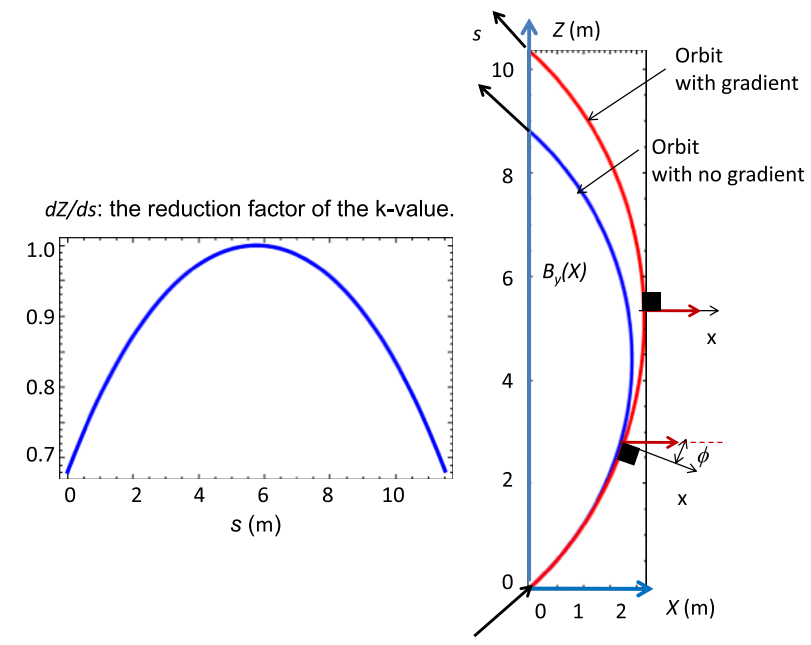

FIG. 8. Orbits of an on-momentum (ideal) particle in the bending magnet with linear field gradient for the case of $B \rho=$ $9[\mathrm{Tm}]$ or $V=38 \mathrm{MV}$ discussed in Sec. IV D, which are obtained by numerically solving equations of motion given in Appendix B.

\section{Reversed field strip}

The roles of the reversed field strip are to introduce large focusing in the vertical direction by reverse bending and to mitigate defocusing effects at the entrance and exit of the main bending field region by introducing a larger injection and extraction angle. This is illustrated in the orbits in Fig. 8, which reflects these angles being increased from 45 degrees to 47.12 degrees.

\section{B. Variable orbit circumference}

The ring circumference is written as

$$
C_{0}=2 \cdot\left(L_{1}+L_{2}+2 \cdot \ell_{B}-2 \tilde{R}\right),
$$

where $L_{1}, L_{2}$, and $\tilde{R}$ are defined in Fig. $1(\mathrm{~b})$, and $\ell_{B}$ is the path length through a single bending magnet, as defined in Appendix B. $C_{0}$ varies with acceleration, schematically shown in Fig. 9.

\section{Lattice and stability analysis}

The proposed ring has mirror symmetry. The symmetry points on the straight sections of the RAFFIA are defined here. The transfer matrix from one point of symmetry to the other is easily written, even including all of the focusing and defocusing components stated above. Although all drift space lengths are parameters subject to optimization, appropriate lengths are assumed here for a preliminary study, where these lengths take into account their availability for injection and extraction devices and acceleration devices. Thus, the only free parameters are the $k$-values of focusing and defocusing quadrupole magnets, that is, $\mathrm{KF}$ and $\mathrm{KD}$, which are defined in the form of $(1 / B \rho) \cdot \partial B_{y} / \partial x$.

Meanwhile, the motion in a bending magnet with a field gradient is a bit complicated. The betatron equations in the horizontal and vertical directions there are described in the following forms:

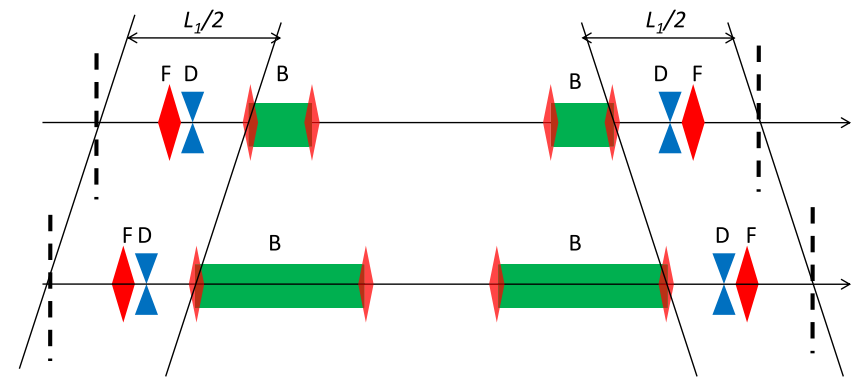

FIG. 9. Half lattice of RAFFIA (upper and lower: in an early/ middle stage of acceleration, respectively), where FD and DF are doublets and $\mathrm{B}$ represents the bending magnet. 


$$
\begin{aligned}
& x^{\prime \prime}(s)+\left[\frac{1}{\rho^{2}(s)}+K(s)\right] x=0, \\
& y^{\prime \prime}(s)-K(s) y=0 .
\end{aligned}
$$

It is noted that the bending radius in the bending magnet varies with $s$,

$$
\begin{aligned}
\frac{1}{\rho(s)} & =\left(\frac{Q}{A}\right)\left(\frac{e}{m c^{2}}\right)\left(\frac{c}{\beta \gamma}\right) B_{y}(s) \\
& =\frac{1}{B \rho} \cdot\left[B_{y}(0)+\frac{\partial B_{y}(X)}{\partial X} \cdot X(s)\right] .
\end{aligned}
$$

Thus, the restoring coefficients of Eq. (4) in this region are given by

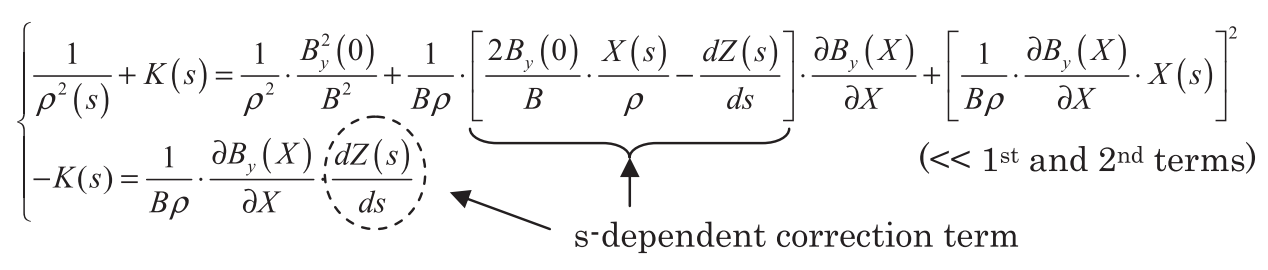

Since the orbit of the ideal particle $X(s)$ and $Z(s)$ on the median plane of the bending magnet is known by numerically solving Eq. (B1), the restoring coefficients are exactly given as functions of $s$. Choosing the orthogonal vectors $x_{1}(0)=(1,0)$ and $x_{2}(0)=(0,1)$ in the phase space $\left(x, x^{\prime}\right)$ or $\left(y, y^{\prime}\right)$ as the initial conditions, solving Eq. (4) to numerically obtain the solutions at the exit of the bending magnet, and employing these solutions $x_{1}\left(\ell_{B}\right)$ and $x_{2}\left(\ell_{B}\right)$, we can write the transfer matrix for the bending magnet as follows:

$$
\begin{aligned}
& M_{B}^{x} \equiv\left(\begin{array}{ll}
m_{11}^{x} & m_{12}^{x} \\
m_{21}^{x} & m_{22}^{x}
\end{array}\right)=\left(\begin{array}{ll}
x_{1}\left(\ell_{B}\right) & x_{2}\left(\ell_{B}\right) \\
x_{1}^{\prime}\left(\ell_{B}\right) & x_{2}^{\prime}\left(\ell_{B}\right)
\end{array}\right) \text { and } \\
& M_{B}^{y} \equiv\left(\begin{array}{ll}
m_{11}^{y} & m_{12}^{y} \\
m_{21}^{y} & m_{22}^{y}
\end{array}\right)=\left(\begin{array}{ll}
y_{1}\left(\ell_{B}\right) & y_{2}\left(\ell_{B}\right) \\
y_{1}^{\prime}\left(\ell_{B}\right) & y_{2}^{\prime}\left(\ell_{B}\right)
\end{array}\right),
\end{aligned}
$$

where $l_{B}$ is the path length in the bending magnet and calculated in Appendix B. Using the transfer matrices for entire focusing effects along the orbit including the edge focusing, we are able to look for a stable region in the
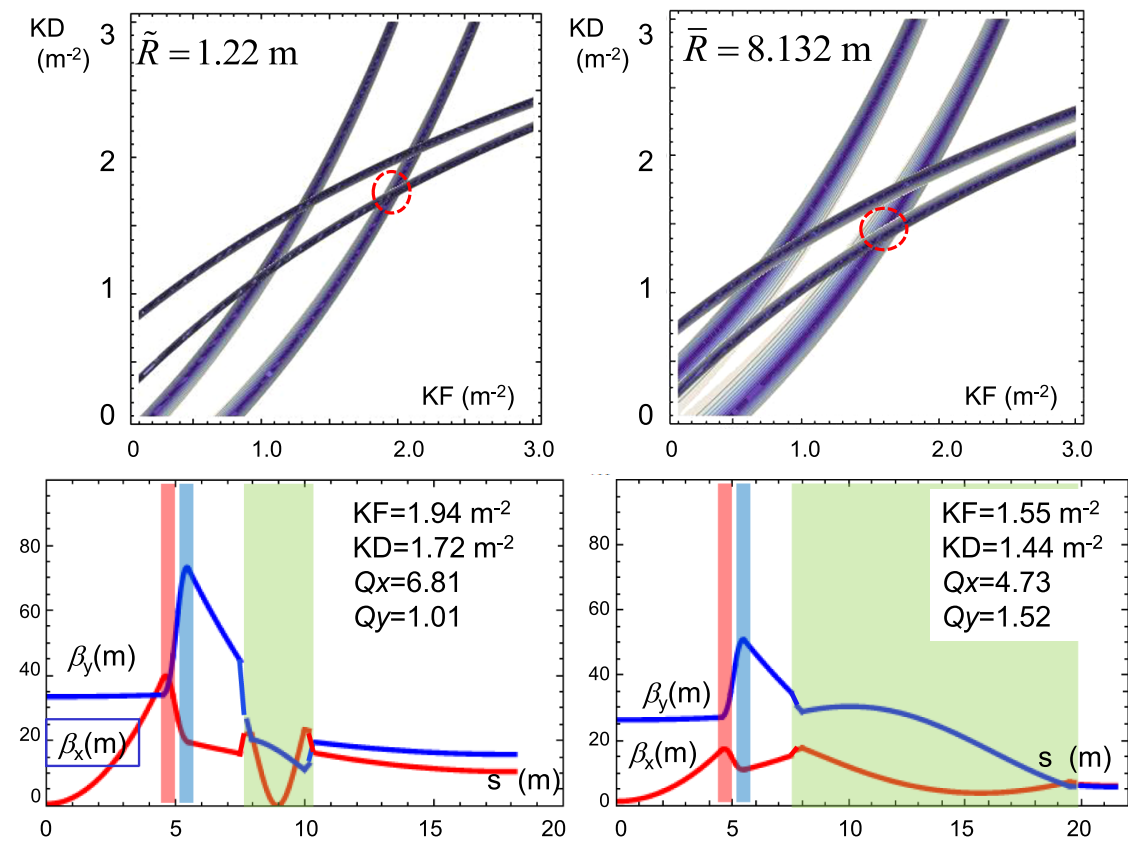

FIG. 10. Upper: Stable regions in the parameter space. Lower: Beta functions for $\mathrm{x}, \mathrm{y}$ directions. Left is for $\tilde{R}=1.22 \mathrm{~m}(B \rho=1.5 \mathrm{Tm}$ or $V=1 \mathrm{MV})$ and right is for $\tilde{R}=8.132 \mathrm{~m}(B \rho=9.0 \mathrm{Tm}$ or $V=38 \mathrm{MV})$. Positions of QF, QD, and bending magnet are shown by the background color of red, blue, and green in the lower figures. 
$K F-K D$ space, where the stability condition is satisfied. The stable regions for the horizontal and vertical direction are shown in the upper plots of Fig. 10. Overlapping regions give stability.

This stability region is found for different energies or magnetic rigidity. The regions' magnitudes gradually change, depending on energy. In other words, tracking is required during one acceleration cycle. It is emphasized that this is a point of difference from a synchrotron, where the $k$-values of focusing and defocusing magnets are usually unchanged. It depends on the capability of power supplies for the doublet how fast the tracking can be realized. Fortunately, acceleration in the RAFFIA is fully maneuvered by the SPS trigger control, as explained in the next section. For instance, the output voltage can be reduced or a number of induction cells in operation is reduced so as to meet a possible tracking speed. Thus, an actual acceleration cycle or acceleration pattern is determined by the capability of the doublet focusing system, especially its power supply.

\section{Lattice function}

When the parameters of the doublet are chosen to give a stable region, the beta function is evaluated to give useful information about the beam envelope. For effective bending radii $\tilde{R}=1.22 \mathrm{~m}$ and $8.312 \mathrm{~m}$, the beta function is also shown in Fig. 10. It is notable that the beta functions vary greatly in the orbit transition region, which variation is caused by dynamic changes in the lattice component parameters.

It is noted that this lattice has a large betatron tune in the horizontal direction. In addition, one finds that the betatron tunes dynamically vary with acceleration in both directions, crossing integer and half-integer tunes. This feature is similar in other fixed field accelerators. Emittance blowup associated with integer and half-integer resonance crossing should be inversely proportional to a speed of acceleration. Thus its size may be determined by the ramping speed of the quadrupole doublet rather than the acceleration voltage, since the induction acceleration voltage is still affordable beyond $50 \mathrm{kV} /$ turn assumed here.

\section{ACCELERATION AND STABILITY IN THE LONGITUDINAL DIRECTION}

\section{A. Acceleration}

As stated in the Introduction, acceleration of cluster ions is provided by acceleration voltage pulses $V_{\text {acc }}$. Triggering of ICs for acceleration must be synchronized with the operation of the doublet, which is excited in a programmed manner. This information is imported into the field programmable gate array (FPGA) code used to generate gate signals [29]. Since the dispersion function is zero over the entirety of the straight section, the position monitor in that section does not help to realize synchronization. $\Delta \mathrm{R}$ feedback control on the basis of the position monitor signals and the digital signal

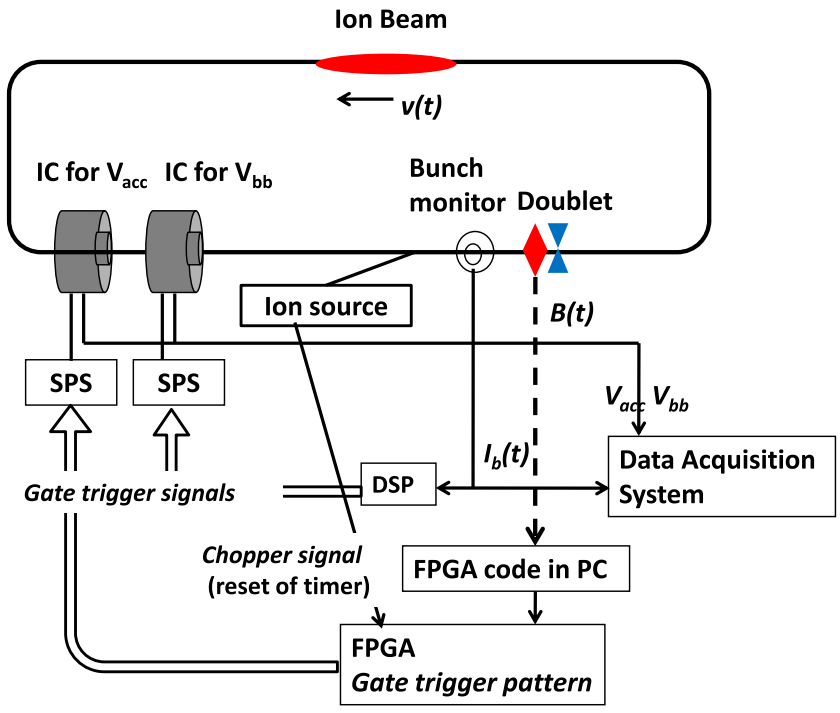

FIG. 11. Control block diagram of the induction acceleration system.

processor control method of the SPS, which was very effective in the slow-cycling induction synchrotron [3], is not available. Precise trigger timing will be determined from the bunch monitor signals, which signal the precise position of the captured ion bunch over time. An outline of the control system is shown in Fig. 11.

In the case of C-60 acceleration, an integrated acceleration voltage of $12-30 \mathrm{kV} /$ turn is assumed. Ten ICs with an output voltage of $3 \mathrm{kV}$ will be utilized.

\section{B. Confinement}

Longitudinal-direction beam confinement in the RAFFIA is provided by barrier voltage pulses. This confinement technique is the same as used in the induction synchrotron. The barrier voltage pulses $V_{b b}$ are generated in the IC for this purpose. Trigger timing of the SPS driving the ICs is gradually varied with acceleration or revolution frequency. The revolution frequency $f$ changes from 8 to $115 \mathrm{kHz}$ for $\mathrm{C}-60$. Bunch confinement is schematically

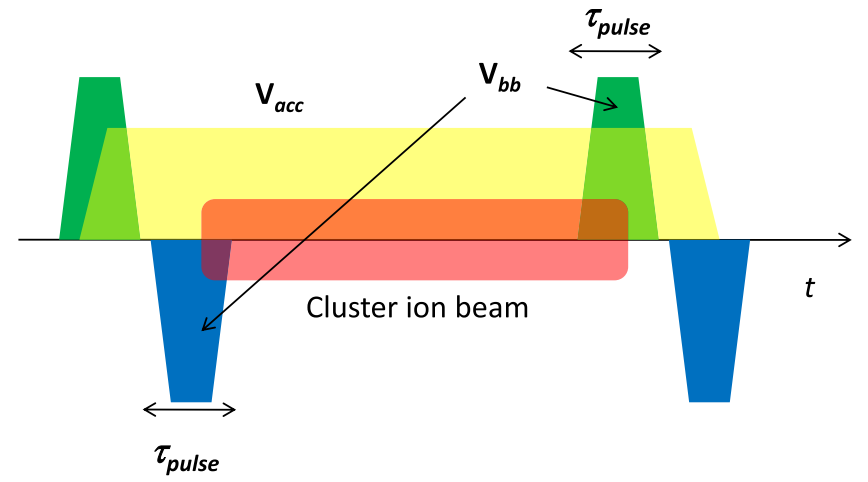

FIG. 12. Barrier voltage pulses for confinement, acceleration voltage pulse, and trapped ion bunch. 
illustrated in Fig. 12, together with the acceleration pulse voltage profile $V_{\text {acc }}$ and a trapped ion bunch.

The required confinement voltage is given by the following equation [8]:

$$
\begin{aligned}
V_{b b}= & \frac{1}{2}\left(\frac{m c^{2}}{e}\right)\left(\frac{T_{0}}{\tau_{\text {pulse }}}\right) \cdot\left(\frac{A}{Q}\right) \cdot \beta_{s}^{2} \cdot \gamma_{s} \cdot\left|\frac{1}{\gamma_{T}^{2}}-\frac{1}{\gamma_{s}^{2}}\right| \\
& \cdot\left(\frac{\Delta p}{p}\right)_{\max }^{2} \cdot
\end{aligned}
$$

Here, $m c^{2}$ is the rest mass energy of proton, $T_{0}$ is the revolution period of the synchronous particle in sec, $\tau_{\text {pulse }}$ is the pulse length of barrier voltage in time, the parameters with the subscript $s$ represent the relativistic $\beta$ and $\gamma$ of the synchronous particle or ideal particle, $\gamma_{T}$ is the time-dependent transition energy, and $(\Delta p / p)_{\max }$ is the maximum momentum spread that can be confined. For simplicity, this magnitude at injection will be roughly estimated as follows. It is assumed that cluster ions are injected into the RAFFIA with an initial energy of $Q e V_{0}$, in which $V_{0}$ is the acceleration voltage of preinjector. Now it is $200 \mathrm{kV}$. Because of $\gamma_{s} \sim 1$ and $\gamma_{T}>1$, further, Eq. (8) reduces to

$$
V_{b b} \leq V_{0} \cdot\left(\frac{T_{0}}{\tau_{\text {pulse }}}\right) \cdot\left(\frac{\Delta p}{p}\right)_{\text {max }}^{2} .
$$

Assuming $T_{0} / \tau_{\text {pulse }} \sim 100$ and $(\Delta p / p)_{\max } \sim 10^{-2}$ as are typical in a conventional circular ring, we obtain $V_{b b} \leq 2 \mathrm{kV}$. This number is quite a nominal value.

\section{Other beam handling}

The combination of induction acceleration, static bending magnets, and programmed operation of doublets allows several distinctive operation modes of the RAFFIA, which are listed below. Group (iii) in particular cannot be realized by rf accelerators: (i) extraction at an arbitrary energy below the maximum energy; (ii) storage of an ion beam at an arbitrary energy; (iii) adiabatic bunch squeezing, dividing, and merging at arbitrary energy. Group (iii) has been already demonstrated in the KEK digital accelerator $[30,31]$.

\section{APPLICATION TO SINGLE-ATOM IONS OR CHARGED MICROPARTICLES}

The RAFFIA is suitable for accelerating singly-charged atomic ions and charged microparticles in a pulsed mode. It is interesting to evaluate the maximum energy per nucleon as a function of $A / Q$, assuming the parameters of the proposed RAFFIA, that is, $B \rho=10.5$. As shown in Fig. 13, the obtainable energy is quite attractive and far beyond that of any cyclotron in operation except for the Superconducting Ring Cyclotron at RIKEN [32]. It is noted
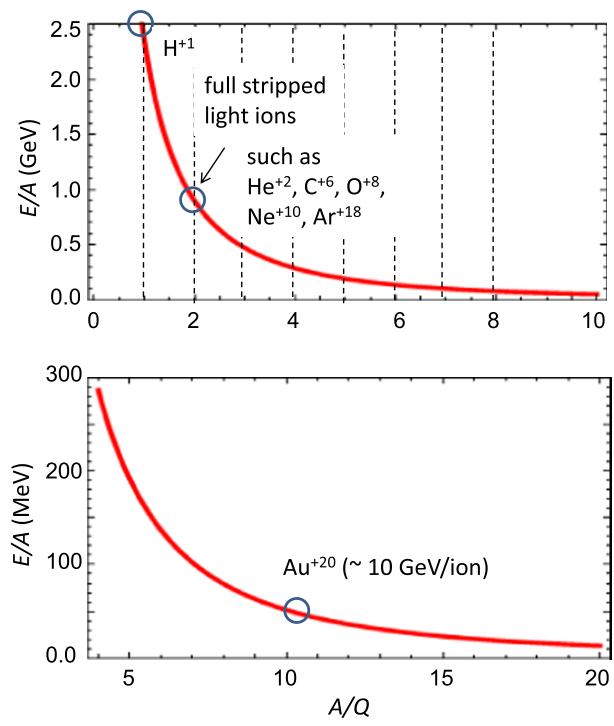

FIG. 13. Obtainable maximum energy per nucleon vs $A / Q$.

here that the RAFFIA will provide necessary ions at any energy below its maximum energy, as shown in the graph.

In practice, the maximum magnetic fields for extremely low $A / Q$ ions such as protons or fully stripped light ions may be reduced a bit at the expense of output energy. The orbit stability at the injection stage, where the bending radius is too small, has not been verified yet. The RAFFIA is essentially a pulsed machine and its acceleration cycle should be a few tens of Hz. The averaged beam current is limited due to this low acceleration cycle. CW cyclotrons that are in operation for applications demanding large averaged beam currents are unable to be replaced by the RAFFIA.

\section{SUMMARY}

A novel RAFFIA capable of accelerating cluster ions by induction acceleration has been proposed, with a design based on our broad experience in the development of the induction synchrotron and our design work on the induction sector cyclotron [7]. The large dynamic range of acceleration frequencies arising from the intrinsic nature of the RAFFIA has been described for an example design. Possible uses for a RAFFIA have been described. This may be a new dawn in both cluster-ion science and accelerator science, opening vistas that nobody has yet dreamed about. Certainly, a RAFFIA would expand the frontiers of materials science that employs giant cluster ions such as C-60 or Si-106 with a super lattice structure [33].

Orbit stability is crucial. There are a lot of concerns on the beam guiding field uniformity. It is practically important to confirm tolerances of imperfection for their key parameters. Hereafter, numerical studies employing the complete 3D simulation capable of handling effects of field imperfections and acceleration/confinement over the entire 
acceleration period are required beyond the present proposal.

\section{ACKNOWLEDGMENTS}

The present study was inspired by Dr. Shinozuka's (Tohoku University Radio Isotope Cyclotron Center) strong interest in a RAFFIA.

\section{APPENDIX A: INTRINSIC EDGE FOCUSING IN A FLAT BENDING MAGNET}

$K$ is a $k$-value of intrinsic weak focusing in a bending magnet, $b$ is the equivalent distance of linear field ramping, and $\theta$ is the entrance and exit angle. $\rho_{\text {ent }}$ is the bending radius at entrance and exit of the bending magnet and $\theta$ is the entrance and exit angle.

\section{APPENDIX B: PATH LENGTH IN A BENDING MAGNET WITH A LINEAR FIELD GRADIENT}

\section{Equations of motion in a bending magnet}

Equations of motion in the $X-Z$ coordinates in Fig. 8 are written by

$$
\begin{aligned}
& \left\{\begin{array}{l}
A \cdot m \gamma \cdot \frac{d^{2} X}{d t^{2}}=Q e \cdot v_{Z} \cdot B_{y} \\
A \cdot m \gamma \cdot \frac{d^{2} Z}{d t^{2}}=-Q e \cdot v_{X} \cdot B_{y}
\end{array}\right. \\
& \Rightarrow\left\{\begin{array}{l}
\frac{d^{2} X}{d t^{2}}=k \cdot v_{Z} \cdot B_{y} \\
\frac{d^{2} Z}{d t^{2}}=-k \cdot v_{X} \cdot B_{y}
\end{array}\right. \\
& \Rightarrow\left\{\begin{array}{l}
\frac{d^{2} X}{d t^{2}}=k \cdot \frac{d Z}{d t} \cdot\left[B_{y}(0)+B_{y}^{\prime}(0) \cdot X\right] \\
\frac{d^{2} Z}{d t^{2}}=-k \cdot \frac{d X}{d t} \cdot\left[B_{y}(0)+B_{y}^{\prime}(0) \cdot X\right]
\end{array},\right.
\end{aligned}
$$

where

$$
\begin{aligned}
B_{y}(X) & =B_{y}(0)+B_{y}^{\prime}(0) \cdot X, & B_{y}^{\prime}(0) \equiv \frac{\partial B_{y}(X)}{\partial X}, \\
k & \equiv\left(\frac{Q}{A}\right)\left(\frac{e}{m c^{2}}\right) \cdot \frac{c^{2}}{\gamma}, & v_{X}^{2}+v_{Z}^{2}=v_{s}^{2}=\text { const. }
\end{aligned}
$$

Integrating the second equation of (B1), $\frac{d^{2} Z}{d t^{2}}=-k \cdot \frac{d X}{d t}$. $\left[B_{y}(0)+B_{y}^{\prime}(0) \cdot X\right]=-k \cdot B_{y}(0) \cdot \frac{d X}{d t}-\frac{k}{2} \cdot B_{y}^{\prime}(0) \frac{d X^{2}}{d t}, \quad$ we have

$$
\frac{d Z}{d t}=-k \cdot B_{y}(0) \cdot X-\frac{k}{2} \cdot B_{y}^{\prime}(0) X^{2}+v_{Z}(0)
$$

Substituting (B2) into the first equation of (B1) we obtain the nonlinear equation for $X$,

$$
\begin{aligned}
\frac{d^{2} X}{d t^{2}}= & k \cdot \frac{d Z}{d t} \cdot\left[B_{y}(0)+B_{y}^{\prime}(0) \cdot X\right] \\
= & k\left\{-k \cdot B_{y}(0) \cdot X-\frac{k}{2} \cdot B_{y}^{\prime}(0) X^{2}+v_{Z}(0)\right\} \\
& \cdot\left[B_{y}(0)+B_{y}^{\prime}(0) \cdot X\right]=k \cdot v_{Z}(0) \cdot B_{y}(0) \\
& +\left[-k^{2} \cdot B_{y}^{2}(0)+k \cdot v_{Z}(0) \cdot B_{y}^{\prime}(0)\right] \cdot X-\frac{3 k^{2}}{2} \\
& \cdot B_{y}^{\prime}(0) \cdot B_{y}(0) \cdot X^{2}-\frac{k^{2}}{2} \cdot\left[B_{y}^{\prime}(0)\right]^{2} \cdot X^{3} .
\end{aligned}
$$

Multiplying both sides of (B3) by $d X / d t(\equiv \dot{X})$ and integrating it with respect to $t$ we have

$$
\begin{aligned}
\dot{X} \cdot \ddot{X} & =\left[k \cdot v_{Z}(0) \cdot B_{y}(0)\right] \cdot \dot{X}+\left[-k^{2} \cdot B_{y}^{2}(0)+k \cdot v_{Z}(0) \cdot B_{y}^{\prime}(0)\right] \cdot X \cdot \dot{X}-\frac{3 k^{2}}{2} \cdot B_{y}^{\prime}(0) \cdot B_{y}(0) \cdot X^{2} \cdot \dot{X}-\frac{k^{2}}{2} \cdot\left[B_{y}^{\prime}(0)\right]^{2} \cdot X^{3} \cdot \dot{X} \\
\frac{1}{2} \frac{d \dot{X}^{2}}{d t} & =\left[k \cdot v_{Z}(0) \cdot B_{y}(0)\right] \cdot \frac{d X}{d t}+\frac{1}{2}\left[-k^{2} \cdot B_{y}^{2}(0)+k \cdot v_{Z}(0) \cdot B_{y}^{\prime}(0)\right] \cdot \frac{d X^{2}}{d t}-\frac{k^{2}}{2} \cdot B_{y}^{\prime}(0) \cdot B_{y}(0) \cdot \frac{d X^{3}}{d t}-\frac{k^{2}}{8} \cdot\left[B_{y}^{\prime}(0)\right]^{2} \cdot \frac{d X^{4}}{d t} \\
\dot{X}^{2} & =2\left[k \cdot v_{Z}(0) \cdot B_{y}(0)\right] \cdot X+\left[-k^{2} \cdot B_{y}^{2}(0)+k \cdot v_{Z}(0) \cdot B_{y}^{\prime}(0)\right] \cdot X^{2}-k^{2} \cdot B_{y}^{\prime}(0) \cdot B_{y}(0) \cdot X^{3}-\frac{k^{2}}{4} \cdot\left[B_{y}^{\prime}(0)\right]^{2} \cdot X^{4}+v_{X}^{2}(0) .
\end{aligned}
$$

Since $\dot{X}=0$ at the return point, it turns out that the return point is the solution of the algebraic equation,

TABLE II. Focusing/defocusing components in the bending magnet.

\begin{tabular}{lll}
\hline \hline & Horizontal & Vertical \\
\hline $\begin{array}{l}\text { Edge focusing both } \\
\text { edges [34] }\end{array}$ & $\left(\begin{array}{cc}1 & 0 \\
\frac{\tan \theta}{\rho_{\mathrm{ent}}} & 1\end{array}\right)\left(\begin{array}{cc}\frac{1}{\rho_{\mathrm{ent}}}\left(\frac{b}{6 \rho_{\mathrm{ent}} \cos \theta}-\tan \theta\right) & 1\end{array}\right)$ \\
\hline \hline
\end{tabular}

$$
\begin{aligned}
& v_{X}^{2}(0)+2\left[k \cdot v_{Z}(0) \cdot B_{y}(0)\right] \\
& \cdot X+\left[-k^{2} \cdot B_{y}^{2}(0)+k \cdot v_{Z}(0) \cdot B_{y}^{\prime}(0)\right] \\
& \cdot X^{2}-k^{2} \cdot B_{y}^{\prime}(0) \cdot B_{y}(0) \cdot X^{3}-\frac{k^{2}}{4} \\
& \quad \cdot\left[B_{y}^{\prime}(0)\right]^{2} \cdot X^{4}=0 .
\end{aligned}
$$

(B5) is numerically evaluated. Assuming that its solution is $X_{\max }$ we can calculate the path length through the bending magnet in the form 


$$
\begin{aligned}
\ell_{B} & =2 \cdot \int_{0}^{X_{\max }} \sqrt{(d X)^{2}+(d Z)^{2}} \\
& =2 \cdot \int_{0}^{X_{\max }} \sqrt{1+\left(\frac{d Z}{d X}\right)^{2}} \cdot d X \\
& =2 \cdot \int_{0}^{X_{\max }} \sqrt{1+\left(\frac{\dot{Z}}{\dot{X}}\right)^{2}} \cdot d X .
\end{aligned}
$$

Here the path length in the reversed field region is ignored.

[1] H. Danmmak, A. Dunlop, D. Lesueur, A. Brunelle, S. Dellanegra, and Y. Le Beyec, Phys. Rev. Lett. 74, 1135 (1995).

[2] 30-MeV C-60 cluster ions (kinetic energy $\sim 0.04 \mathrm{MeV} / \mathrm{u}$ ) have been provided by the $10 \mathrm{MV}$ tandem accelerator of the Institute of Nuclear Physics in Orsay (IPNO). A. E. El-Said, Nucl. Instrum. Methods Phys. Res., Sect. B 267, 953 (2009).

[3] K. Takayama and J. Kishiro, Nucl. Instrum. Methods Phys. Res., Sect. A 451, 304 (2000).

[4] K. Takayama, Y. Arakida, T. Dixit, T. Iwashita, T. Kono, E. Nakamura, K. Otsuka, Y. Shimosaki, K. Torikai, and M. Wake, Phys. Rev. Lett. 98, 054801 (2007).

[5] K. Takayama, T. Yoshimoto, M. Barata, L. K. Wah, L. Xingguang, T. Iwashita, S. Harada, T. Adachi, T. Arai, D. Arakawa, H. Asao, E. Kadokura, T. Kawakubo, H. Nakanishi, Y. Okada, K. Okamura, K. Okazaki, A. Takagi, S. Takano, and M. Wake, Phys. Rev. ST Accel. Beams 17, 010101 (2014).

[6] K. Takayama, Y. Arakida, T. Iwashita, Y. Shimosaki, T. Dixit, and K. Torikai, J. Appl. Phys. 101, 063304 (2007); J. Appl. Phys. 103, 099903(E) (2008); patent in Japan 3896420 (2007); Y. Oguri et al., in Proceedings of RPIA2006, pp. 139-145 (KEK Proceedings 2006-12) (2007); T. Iwashitaet al., Phys. Rev. ST Accel. Beams 14, 071301 (2011).

[7] K. Takayama, T. Adachi, H. Tsutsui, W. Jiang, and Y. Oguri, in Proceedings of the 19th International Conference on Cyclotrons and their Applications in China, Lanzhou, 2010, pp. 331-333.

[8] K. Takayama, Induction Accelerators, edited by K. Takayama and R. Briggs (Springer, New York, 2010), Chap. 11.

[9] V. I. Veksler, Dokl. Akad. Nauk USSR 43, 329 (1944).

[10] J. Itoh and D. Kobayashi, Kagaku 17, 34 (1947).

[11] J. S. Schwinger, Lectures at the Los Alamos laboratory at the end of July 1945 (1945); K. A. Miton and J.S. Schwinger, Electromagnetic Radiatation: Variational
Methods, Waveguides and Accelerators (Springer, Berlin, 2006), p. 265.

[12] L. I. Schiff, Rev. Sci. Instrum. 17, 6 (1946).

[13] P. A. Redhead, H. LeCaine, and W. J. Henderson, Nucleonics 5, 60 (1949).

[14] A. Roberts, Ann. Phys. (Paris) 4, 115 (1958).

[15] K. H. Kaiser et al., Nucl. Instrum. Methods Phys. Res., Sect. A 593, 159 (2008).

[16] H. Herminghaus, K. H. Kaiser, and U. Ludwig, Nucl. Instrum. Methods 187, 103-107 (1981); R. E. Rand, Recirculating Electron Accelerators (Harwood Academic Publishers, New York, 1984), p. 149.

[17] V. R. Bhardwaj, P. B. Corkum, and D. M. Rayner, Phys. Rev. Lett. 91, 203004 (2003).

[18] T. Adachi, T. Arai, K. W. Leo, K. Takayama, and A. Tokuchi, Rev. Sci. Instrum. 82, 083305 (2011).

[19] K. W. Leo, T. Adachi, T. Arai, and K. Takayama, Phys. Rev. ST Accel. Beams 16, 043502 (2013).

[20] T. Adachi and T. Kawakubo, Phys. Rev. ST Accel. Beams 16, 053501 (2013).

[21] H. Kobayashi, T. Kawakubo, and A. Tokuchi, in Proceedings of the 5th Euro-Asian Pulsed Power Conference Kumamoto, Japan (2014).

[22] M. Wake et al., in Proceedings of the 22nd Particle Accelerator Conference, PAC-2007, Albuquerque, NM (IEEE, New York, 2007), pp. 251-253.

[23] K. Okamura, K. Ise, M. Wake, K. Takaki, Y. Osawa, and K. Takayama, IEEE Trans. Plasma Sci. 40, 2205 (2012).

[24] K. Okamura, Y. Ohsawa, M. Wake, T. Yoshimoto, R. Sasaki, K. Takaki, and K. Takayama, Mater. Sci. Forum 778-780, 883 (2014).

[25] T. Tanabe and K. Noda, Nucl. Instrum. Methods Phys. Res., Sect. A 496, 233 (2003).

[26] H. Sato, in Proceedings of the 18th Particle Accelerator Conference, New York, 1999 (IEEE, New York, 1999), pp. 2650-2652.

[27] J. Jankowiak, Eur. Phys. J. A 28, 149 (2006).

[28] I. Yamane (private communication).

[29] S. Harada and Y. Barata, Master thesis, Tokyo City University, 2011-2012.

[30] L. Xiggung et al., The 3rd International Workshop on Recent Progress in Induction Accelerators, Chengdu, China, 2011.

[31] T. Yoshimoto et al., in Proceedings of IPAC2014 (Dresden, Germany, 2014), p. 1893.

[32] H. Okuno, J. Ohnishi, K. Yamada, N. Fukunishi, K. Ikegami, T. Maie, H. Hasebe, M. Hamanaka, M. Kase, A. Goto, and Y. Yano, IEEE Trans. Appl. Supercond. 17, 1063 (2007).

[33] Y. Iwata, K. Tomita, T. Uchida, and H. Matsuhata, Cryst. Growth Des. 15 (2015).

[34] C. Bovet, R. Gouiran, I. Gumowski, and K. H. Reich, CERN Report No. CERN/MPS-SI/Int. DL/70/4. 